\title{
Effectiveness of dapagliflozin on vascular endothelial function and glycemic control in patients with early-stage type 2 diabetes mellitus: DEFENCE study
}

Fumika Shigiyama ${ }^{1}$, Naoki Kumashiro ${ }^{1^{*}} \mathbb{0}$, Masahiko Miyagi ${ }^{1}$, Kayoko Ikehara $^{1}$, Eiichiro Kanda ${ }^{2,3}$, Hiroshi Uchino ${ }^{1}$ and Takahisa Hirose ${ }^{1}$

\begin{abstract}
Background: Recent studies reported that sodium glucose cotransporter 2 (SGLT2) inhibitors can potentially reduce the risk of cardiovascular mortality in patients with type 2 diabetes mellitus (T2DM). However, there is little or no information on the therapeutic effects of SGLT2 inhibitors on the progression of atherosclerosis. This dapagliflozin effectiveness on vascular endothelial function and glycemic control (DEFENCE) study was designed to determine the effects of dapagliflozin, a SGLT2 inhibitor, on endothelial function in patients with early-stage T2DM.

Methods: DEFENCE is a prospective, randomized, open-label, blinded-endpoint, parallel-group, comparative clinical trial. Between October 2015 and August 2016, 80 T2DM patients treated with 750 mg of metformin (hemoglobin A1c $\geq 6.0$ and $<8.0 \%, \mathrm{n}=80$ ) were enrolled and randomized to receive either $1500 \mathrm{mg} /$ day metformin (the metformin group, $n=40$ ), or $750 \mathrm{mg} /$ day metformin supplemented with $5 \mathrm{mg}$ /day dapagliflozin (the dapagliflozin group, $n=40$ ), for 16 weeks. The primary endpoint was a change in flow-mediated dilation (FMD) from baseline to the end of the 16-week treatment period. The secondary outcomes include changes in indexes of glycemic control, lipid metabolism, and oxidative stress, body composition, and safety evaluation.

Results: Although FMD tended to improve only in the dapagliflozin group, $\triangle F M D$ was comparable between the two groups. Analysis of patients with $\mathrm{HbA1C}>7.0 \%$ showed significant improvement of FMD in the dapagliflozin group than metformin group $(P<0.05)$. HbA1c, fasting plasma glucose, plasma glucagon, and body weight significantly decreased in both groups. Interestingly, urine 8-hydroxy-2'-deoxyguanosin, a biomarker of oxidative stress, was significantly lower in the dapagliflozin group than metformin group at 16 weeks $(P<0.001)$.

Conclusions: Dapagliflozin add-on therapy to metformin for 16 weeks improved endothelial function, as assessed by FMD, in patients with inadequately controlled early-stage T2DM. Improvement in oxidative stress may contribute to the improvement in FMD.

Trial registration University Hospital Medical Information Network Clinical Trial Registry (UMIN000018754)
\end{abstract}

Keywords: Dapagliflozin, Endothelial function, Type 2 diabetes

\footnotetext{
*Correspondence: naoki.kumashiro@med.toho-u.ac.jp

${ }^{1}$ Division of Diabetes, Metabolism, and Endocrinology, Department

of Medicine, Toho University Graduate School of Medicine, 6-11-1

Omori-Nishi, Ota-ku, Tokyo 143-8541, Japan

Full list of author information is available at the end of the article
} 


\section{Background}

Type 2 diabetes mellitus (T2DM) is a major risk factor for the progression of atherosclerosis and development of cardiovascular diseases $[1,2]$. It is reported that the prevalence of coronary heart disease is two to fourfolds higher in T2DM patients compared to non-diabetes patients [3]. The risk of cardiovascular diseases progressively increases at the stage of impaired glucose tolerance and/or post-prandial hyperglycemia [4,5]. The UKPDS study concluded that treatment of T2DM at an early stage significantly reduces the incidence of macroangiopathy [6]. This is conceivable since it is difficult to improve endothelial function after the progression of atherosclerosis. Thus, early screening for atherosclerosis is important in the prevention of future cardiovascular events. The initial stages of atherosclerosis can be detected at present by using various non-invasive devices. Among them, the flow-mediated dilation (FMD) method can measure endothelial function and predict the prognosis of cardiovascular events [7].

Various oral glucose-lowering agents with different mechanisms of action are currently available in the market. The American Diabetes Association and the European Association for the Study of Diabetes recommend the use of metformin as the first-line drug [8-10]. Metformin has been reported to prevent cardiovascular events in obese patients [8]. Inhibitors of sodium glucose cotransporter 2 (SGLT2), a new family of oral glucoselowering agents, prevent a rise in blood glucose level by suppressing renal reabsorption of sodium and glucose, and by enhancing urinary glucose excretion [11]. Dapagliflozin was the first SGLT2 inhibitor introduced to the world market. SGLT2 inhibitors have additional effects beyond lowering blood glucose, such as helping reduce body weight [12], lower blood pressure [13], and reduce serum triglyceride level [14]. It has also been reported that SGLT2 inhibitors, through their blood pressure lowering effects, can improve arterial stiffness [15]. Thus, SGLT2 inhibitors seem to have multiple metabolic and cardiovascular benefits. However, only a few large cardiovascular outcome studies designed to elucidate the effect of SGLT2 inhibitors have been conducted [16, 17]. These include a currently ongoing clinical trial on dapagliflozin [Dapagliflozin Effect on CardiovascuLAR Events (DECLARE TIMI-58); NCT01730534]. Recently, the EMPA-REG outcomes trial investigated the effects of empagliflozin, another SGLT2 inhibitor [17], and reported that empagliflozin significantly reduced the all-cause and cardiovascular mortality rates and the rate of hospitalization for heart failure compared to placebo in T2DM patients with high cardiovascular risk [17]. Similarly, dapagliflozin may lower blood glucose levels and at the same time prevent cardiovascular events.
The clinical trial regarding a possible favorable action of dapagliflozin on heart failure, the REFORM study [18], is ongoing and we should expect for the results. However, there is no information on whether SGLT2 inhibitors can halt the progression of early atherosclerosis as primary prevention.

The present clinical trial [dapagliflozin effectiveness on vascular endothelial function and glycemic control in T2DM (DEFENCE)] is the first study designed to assess the anti-atherosclerotic effects of dapagliflozin in earlystage T2DM patients, using FMD as a surrogate marker for cardiovascular events. The study also compared the effects of dapagliflozin add-on to those of metformin alone on various metabolic markers.

\section{Methods}

\section{Study design}

The DEFENCE study is a prospective, randomized open-label, blinded-endpoint, parallel-group, comparative study, registered with the University Hospital Medical Information Network Clinical Trial Registry (UMIN000018754), a non-profit organization in Japan that meets the requirements of the International Committee of Medical Journal Editors (ICMJE). The study was approved by the Medical Ethics Committee of Toho University (approval \#27249) and conducted according to the Declaration of Helsinki and current legal regulations in Japan. The processes of enrollment, randomization, data collection and management were conducted by thirdparty entities to secure non-bias.

\section{Study population}

A total of 80 Japanese patients with T2DM who regularly visited the Outpatient Clinics of 15 institutions in Japan (listed under Additional file 1) participated in the study. The inclusion criteria were as follows: (1) type 2 diabetes patients who have been treated for more than 12 weeks with $750 \mathrm{mg}$ of metformin or one type of oral glucoselowering agent in addition to $750 \mathrm{mg}$ of metformin (in the case of sulfonylurea users, $<2 \mathrm{mg}$ of glimepiride or $<40 \mathrm{mg}$ of gliclazide were allowed), in addition to diet and exercise; (2) hemoglobin A1c (HbA1c) (National Glycohemoglobin Standardization Program; NGSP) level of $\geq 6.0$ to $<8.0 \%$; (3) males and females aged 20-74; (4) patients who could be monitored closely for medication compliance; (5) signing written consent form to participate in the study. The following criteria were used to exclude subjects from the study: (1) patients with type 1 diabetes or secondary diabetes; (2) patients who, within 12 weeks before signing the consent form, had used SGLT2 inhibitors, glucagon-like peptide-1 agonists, or insulin; (3) patients who, within 12 weeks before signing the consent, had used a dose of metformin exceeding 
$750 \mathrm{mg} /$ day; (4) patients who, within 12 weeks before consent, had started taking angiotensin-converting enzyme inhibitor (ACE inhibitor), angiotensin II receptor antagonist (ARB), HMG-CoA reductase inhibitor (statin), or antiplatelet drugs, or had the dose changed (including reduction of the dose); (5) patients with severe infection, were scheduled for surgery, or suffered serious trauma recently; (6) patients with history of myocardial infarction, angina, stroke or cerebral infarction; (7) patients with atrial (chronic) fibrillation, frequent supraventricular or ventricular ectopy; (8) patients with moderate or severe cardiac insufficiency (those with class III or more as classified by the NYHA/New York Heart Association); (9) patients with ankle brachial pressure index of $<0.9$; (10) patients with serious liver or renal functional failure [serum creatinine $\geq 1.3 \mathrm{mg} / \mathrm{dL}$ or estimated glomerular filtration rate (eGFR) of $\left.<45 \mathrm{~mL} / \mathrm{min} / 1.73 \mathrm{~m}^{2}\right]$; (11) patients who, within 12 weeks before consent, had unstable blood pressure, or lipid abnormalities; (12) patients dependent on alcohol or illicit drugs; (13) female patients who were pregnant or breastfeeding, possibly pregnant, or planning to become pregnant within the study period; (14) patients with dehydration (abnormal test results of hematocrit and BUN values, and complaint of symptoms of dehydration); (15) patients on diuretics; (16) patients with urinary tract or genital infections within 12 weeks before consent; (17) patients with history of hypersensitivity to the study drugs; (18) patients with severe ketosis, diabetic coma or precoma; (19) patients considered unsuitable subjects by the attending physician; (20) patients with an implanted pacemaker.

\section{Randomization and study intervention}

The eligible subjects were randomly assigned in equal numbers into two groups; the metformin group: the dose of metformin was increased from 750 to $1500 \mathrm{mg} /$ day, and the dapagliflozin group: $5 \mathrm{mg}$ /day dapagliflozin was added to $750 \mathrm{mg} /$ day metformin. The randomization was achieved by a computer-based dynamic allocation method based on the with/without administration of statins and dipeptidyl peptidase 4 (DPP-4) inhibitors. After enrollment in the study, all patients were prohibited from changing the dose of concomitant drugs or adding any other drugs, such as other glucose-lowering agents, anti-hypertension drugs, lipid-lowering and antiplatelet agents. Baseline measurements of blood and urine variables and FMD were performed during the 4-6 weeks of the screening period. After baseline data collection, the assigned therapies were started. The treatment intervention date was set as the study start date (metformin group: the day of increasing the dose of metformin; dapagliflozin group: the day of addition of dapagliflozin to metformin). The assigned treatment was continued for 16 weeks (duration of the study).

\section{Recorded variables and schedule}

Clinical and biochemical data were collected at baseline and after the 16-week treatment period. The FMD was conducted at Toho University Omori Medical Center. All blood tests were carried out after overnight fast. Measurement of the following parameters was outsourced to the central laboratory (SRL Laboratory, Tokyo, Japan): $\mathrm{HbA1c}$, fasting plasma glucose (FPG), C-peptide, plasma insulin, glucagon, total cholesterol, high-density lipoprotein (HDL) cholesterol, low-density lipoprotein (LDL) cholesterol, triglyceride, adiponectin, apolipoprotein B48 and urinary 8-hydroxy-2'-deoxyguanosin (8-OHdG). The homeostatic model assessment of insulin resistance (HOMA-IR) was calculated from the obtained data. In addition to these parameters, other biochemical safety parameters (e.g. red blood cell count, hematocrit, and uric acid) were measured.

\section{Flow-mediated dilation}

The FMD was measured using the UNEX EF38G (UNEX Corporation, Nagoya, Japan) by a technologist who was not a participant in the study and was blinded to the study groups. The protocol and methodology have been described in detail previously [19-21]. Briefly, all measurements were performed under fasting and nonsmoking conditions in the early morning in a temperature-controlled room $\left(25^{\circ} \mathrm{C}\right)$. After resting for at least $15 \mathrm{~min}$, the pressure cuff was placed on the forearm to capture baseline images of the brachial artery using highresolution ultrasound. Then, the cuff was inflated and kept at $50 \mathrm{mmHg}$ above the systolic blood pressure to occlude the brachial artery. The cuff was released $5 \mathrm{~min}$ later, and the image of the brachial artery was captured. The diameters of the brachial artery on the pre- and posthyperemia images were used to calculate changes in FMD according to the following formula: [FMD $(\%)=($ maximum diameter - diameter at rest $) \times 100 /$ diameter at rest].

\section{Study outcome}

The primary study outcome was a change in FMD [ $\triangle$ FMD (=value at week $16-$ value at baseline)]. The secondary endpoints included changes in the values of the following items at the end of the 16-week treatment, relative to the baseline: (1) indexes of glycemic control: HbA1c, FPG level, C-peptide, plasma insulin, glucagon and HOMA-IR; (2) indexes of lipid metabolism: total cholesterol, HDL cholesterol, LDL cholesterol and triglyceride; (3) indexes of atherosclerosis and oxidative 
stress: adiponectin, apolipoprotein B48, and urinary 8-OHdG.

\section{Safety and evaluation of adverse events}

During the course of the study, the investigators constantly monitored the appearance of any hypoglycemic or hyperglycemic symptoms and signs and all other adverse events (AEs) through regular medical checkups. When AEs occurred, details were reported immediately to the respective institution, the principal investigator and the administration office. All related AEs, not only side effects to the drug, but also abnormal values from the clinical tests, were reported and documented.

\section{Sample size and statistical analysis}

Due to the lack of previous reports on $\triangle$ FMD with SGLT2 inhibitor therapy, $\triangle$ FMD under treatment with DPP-4 inhibitor were used as reference for sample size calculation. It has been reported that 12-week treatment with sitagliptin (DPP-4 inhibitor) produced a mean $\triangle \mathrm{FMD}$ of $1.69 \pm 1.76 \%( \pm \mathrm{SD})[22]$. Accordingly, we assumed that a similar $\triangle$ FMD would be expected after 16-week dapagliflozin therapy. Furthermore, another study reported that 24-week metformin therapy produced $\triangle \mathrm{FMD}$ of $0.72 \pm 1.52 \%$ [23]. Accordingly, we assumed that 16 -week metformin treatment in this study would produce $\triangle \mathrm{FMD}$ of $0.5 \pm 1.52 \%$. Thus, we assumed that the difference in $\triangle$ FMD after 16-week treatment between the two groups would be $1.19 \%$. Based on these assumptions, the number of cases required to detect a significant difference in $\triangle$ FMD between the two groups under the conditions of two-sided $P$ value of $5 \%$ and power of $85 \%$, was 36 patients per group; with a total sample size of 72 . Assuming a dropout rate of $10 \%$, the target number of patients was therefore set to 40 cases per group, with a total of 80 cases.

Analyses of the primary and secondary endpoints were performed on the full analysis set (FAS). FAS includes subjects who were enrolled in this study and assigned to a study treatment, however, research subjects with a significant study protocol violation were excluded. Safety analysis with $\mathrm{AE}$ was performed on the treated set. Every subgroup analysis plan was pre-specified before the statistical analysis plan was determined.

The reported values were expressed as mean \pm SD unless otherwise mentioned in the text. Statistical analyses were conducted with two-sided $P$ value set at $5 \%$. Summary statistics were executed for background data. The Fisher's exact test was applied for nominal variables, and the Student's $t$ test was applied for continuous variables for comparisons between groups. The analysis plan resembled that applied in a previous study published by our group [21]. Briefly, for the primary endpoint, i.e., $\triangle F M D$ after 16-week treatment, we compared the fixed effects of the groups using analysis of covariance and covariates of the allocation adjustment factors (statin use and DPP-4i use). Analyses of the secondary endpoints were performed using Student's $t$ test for comparisons of two groups. Regarding safety information, a list of all AEs was prepared for each group and compared using Fisher's exact test. All statistical analyses were performed by the administrative office of the DEFENCE study, with supervision by an independent statistician, using SAS software version 9.3 (SAS Institute Inc., Cary, NC).

\section{Human rights and ethical principles of study subjects}

The study protocol complied with the "World Medical Association Declaration of Helsinki" (2013 revision), and "Ethical Guidelines for Medical and Health Research Involving Human Subjects" (December 22, 2014, Ministry of Education, Culture, Sports, Science and Technology/Ministry of Health, Labor and Welfare), and all other relevant laws and regulations.

\section{Results \\ Clinical characteristics of the two groups}

A total of 80 patients were enrolled in this study between October 2015 and August 2016. They were randomized into the metformin group and the dapagliflozin group. Of the total, 74 patients completed the study and the FAS population included each 37 patients in the metformin group and dapagliflozin group, respectively (Fig. 1). Table 1 shows the baseline clinical characteristics of the patients. There were no significant differences in all the clinical characteristics between both groups. Notably, the mean duration of T2DM was around 6 years and the mean HbA1c was lower than $7 \%$ in both groups. Table 1 shows that only a small number of the study patients had diabetic or macrovascular complications.

\section{Endothelial function after 16 weeks of treatment}

The primary endpoint of this study was the $\triangle \mathrm{FMD}$ after 16 weeks of treatment. Table 2 shows the FMD values at baseline, week 16 and the $\triangle$ FMD values. Although FMD tended to improve in the dapagliflozin group $(\mathrm{P}=0.06)$, $\triangle \mathrm{FMD}$ was comparable between the two groups in the FAS population (Table 2; Fig. 2a). Interestingly, however, subgroup analyses of patients with $\mathrm{HbA} 1 \mathrm{c} \geq 7.0 \%$ for $\triangle F M D$ showed that FMD improved significantly in the dapagliflozin group compared with the metformin group $(\mathrm{P}<0.05$, Table 2; Fig. $2 \mathrm{~b})$. The result of covariance analysis for $\triangle F M D$ after adding the baseline FMD also showed the significant difference between the two groups $(\mathrm{P}<0.05)$. 


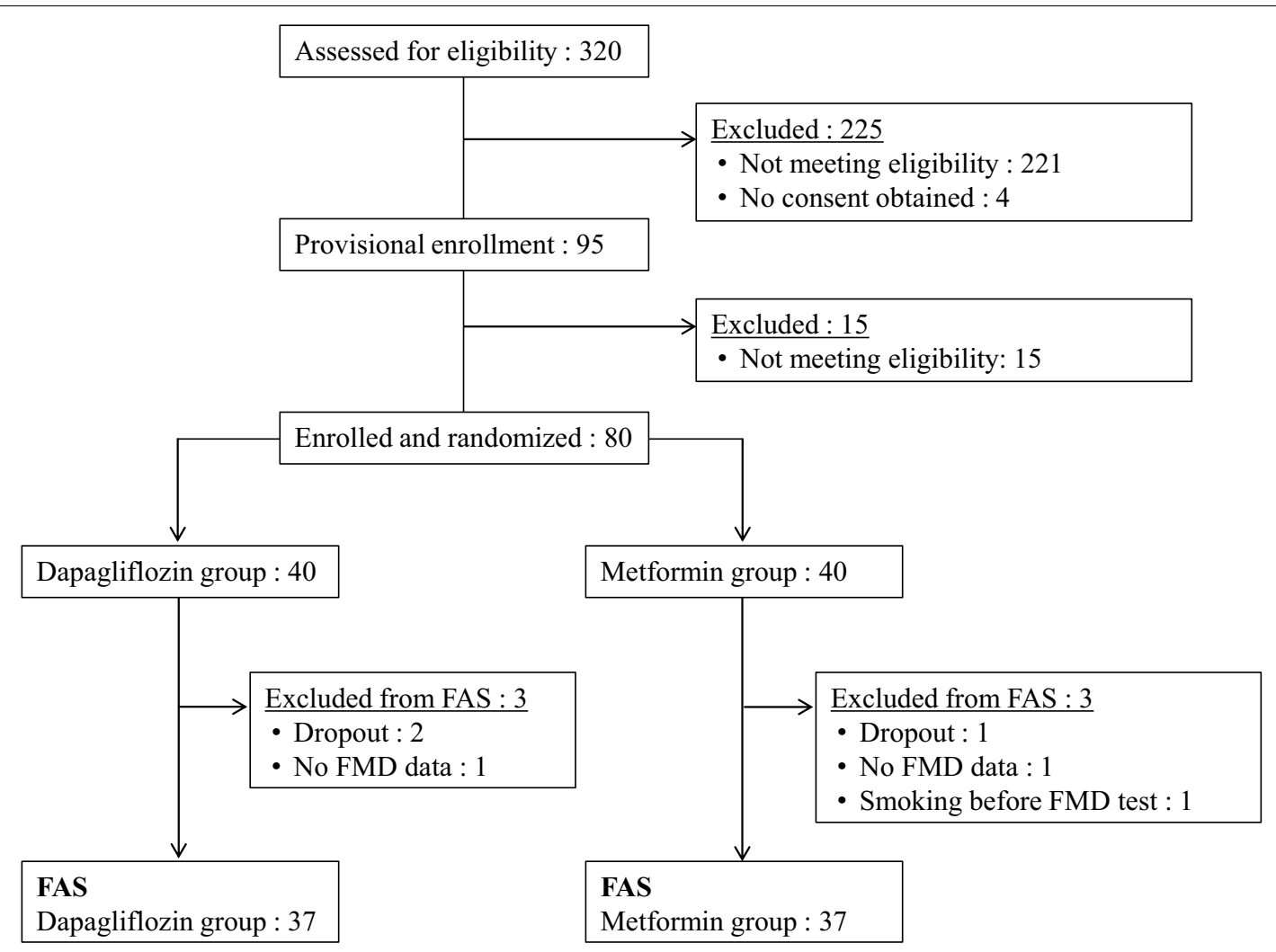

FAS: Full analysis set

Fig. 1 Recruitment process of patients. FAS full analysis set

\section{Comparable changes in glycemic control between the two groups}

HbA1c and fasting plasma glucose improved significantly from baseline to 16-weeks in both groups (Table 3 ). Fasting plasma insulin decreased significantly only in the dapagliflozin group, while plasma glucagon level decreased in both groups. HOMA-IR, which is recognized as an index of insulin resistance, decreased significantly in both groups.

\section{Changes in clinical parameters of atherosclerosis and oxidative stress}

Body weight and BMI decreased significantly in the dapagliflozin group compared with the metformin group $(-1.9 \pm 1.5$ vs. $-0.6 \pm 1.3 \mathrm{~kg} ; \mathrm{P}<0.001$, $-0.7 \pm 0.6$ vs. $-0.2 \pm 0.5 \mathrm{~kg} / \mathrm{m}^{2} ; \mathrm{P}<0.001$, respectively, Additional file 1: Table $\mathrm{S} 1$ ). With regard to lipid metabolism, total cholesterol and non-HDL cholesterol decreased significantly only in the metformin group, HDL cholesterol increased significantly only in the dapagliflozin group, LDL cholesterol increased significantly in both groups (Table 4). Triglyceride did not change in both groups. No significant changes were observed in serum adiponectin and apolipoprotein B48 in both groups. Compared to the metformin group, urine 8-OHdG/creatinine, a marker of oxidative stress, decreased significantly in the dapagliflozin group (Table 4).

\section{Metabolic and hemodynamic changes}

Significant increases in red blood cell count, hemoglobin, and hematocrit were noted in the dapagliflozin group (Table 5). In contrast to the above dynamic changes, white blood cell count remained unchanged, and platelet count decreased slightly in the dapagliflozin group and increased in the metformin group. Plasma uric acid decreased in the dapagliflozin group and was significantly different between the two groups $(\mathrm{P}<0.001$, Table 5$)$. Analysis of data of patients with $\mathrm{HbA} 1 \mathrm{c} \geq 7.0 \%$ showed significant increases in red blood cell count, hemoglobin, and hematocrit and decrease in plasma uric acid in the dapagliflozin group (Table 6). Systolic and diastolic blood pressure were comparable between the two groups (Table 4). 
Table 1 Patients' characteristics

\begin{tabular}{|c|c|c|c|}
\hline Characteristics & $\begin{array}{l}\text { Dapagliflozin } \\
\text { group }\end{array}$ & $\begin{array}{l}\text { Metformin } \\
\text { group }\end{array}$ & $P$ value \\
\hline Sex (male/female) & $25(67.6) / 12(32.4)$ & $22(59.5) / 15(40.5)$ & 0.63 \\
\hline Age (years) & $57.9 \pm 8.3(37)$ & $59.4 \pm 10.1(37)$ & 0.51 \\
\hline $\begin{array}{l}\text { Duration of diabetes } \\
\text { (years) }\end{array}$ & $5.4 \pm 4.4(37)$ & $6.3 \pm 4.2(37)$ & 0.40 \\
\hline $\operatorname{BMI}\left(\mathrm{kg} / \mathrm{m}^{2}\right)$ & $26.8 \pm 4.6(37)$ & $26.3 \pm 3.5(37)$ & 0.60 \\
\hline Current smoking & $10(27.0)$ & $6(16.2)$ & 0.50 \\
\hline Current alcohol & $14(37.8)$ & $12(32.4)$ & 0.81 \\
\hline Diabetic retinopathy & $6(16.2)$ & $2(5.4)$ & 0.26 \\
\hline Simple retinopathy & $4(10.8)$ & $0(0.0)$ & 0.09 \\
\hline $\begin{array}{l}\text { Preproliferative } \\
\text { retinopathy }\end{array}$ & $1(2.7)$ & $0(0.0)$ & \\
\hline $\begin{array}{l}\text { Proliferative retin- } \\
\text { opathy }\end{array}$ & $1(2.7)$ & $2(5.4)$ & \\
\hline $\begin{array}{l}\text { Diabetic nephropa- } \\
\text { thy }\end{array}$ & $10(27.0)$ & $13(35.1)$ & 0.62 \\
\hline $\begin{array}{l}\text { UACR } \\
\text { 30-299 mg/g } \\
\text { creatinine }\end{array}$ & $9(24.3)$ & $13(35.1)$ & 0.45 \\
\hline $\begin{array}{l}\text { UACR } \geq 300 \mathrm{mg} / \mathrm{g} \\
\text { creatinine }\end{array}$ & $1(2.7)$ & $0(0.0)$ & \\
\hline Diabetic neuropathy & $6(16.2)$ & $6(16.2)$ & 1.00 \\
\hline Polyneuropathy & $3(8.1)$ & $0(0.0)$ & 0.13 \\
\hline Mononeuropathy & $1(2.7)$ & $5(13.5)$ & \\
\hline Unknown & $2(5.4)$ & $1(2.7)$ & \\
\hline $\begin{array}{l}\text { Macrovascular } \\
\text { complications }\end{array}$ & $1(2.7)$ & $1(2.7)$ & 1.00 \\
\hline $\begin{array}{l}\text { Cerebrovascular } \\
\text { disease }\end{array}$ & $0(0.0)$ & $0(0.0)$ & - \\
\hline Coronary disease & $0(0.0)$ & $0(0.0)$ & - \\
\hline $\begin{array}{l}\text { Peripheral arterial } \\
\text { disease }\end{array}$ & $1(2.7)$ & $1(2.7)$ & 1.00 \\
\hline Other complications & $34(91.9)$ & $32(86.5)$ & 0.71 \\
\hline Renal disease & $1(2.7)$ & $0(0.0)$ & 1.00 \\
\hline Liver disease & $4(10.8)$ & $3(8.1)$ & 1.00 \\
\hline Hypertension & $15(40.5)$ & $14(37.8)$ & 1.00 \\
\hline Hyperlipidemia & $25(67.6)$ & $23(62.2)$ & 0.81 \\
\hline HbA1c (NGSP\%) & $6.8 \pm 0.5(37)$ & $6.9 \pm 0.5(37)$ & 0.39 \\
\hline $\mathrm{HbA} 1 \mathrm{c}(\mathrm{mmol} / \mathrm{mol})$ & $50.3 \pm 5.5(37)$ & $51.4 \pm 5.7(37)$ & 0.39 \\
\hline $\begin{array}{l}\text { Fasting plasma } \\
\text { glucose (mg/dL) }\end{array}$ & $133.5 \pm 27.1(37)$ & $139.6 \pm 20.2(37)$ & 0.28 \\
\hline C-peptide (ng/mL) & $2.1 \pm 0.8(37)$ & $2.3 \pm 1.1(37)$ & 0.34 \\
\hline Insulin $(\mu \mathrm{IU} / \mathrm{mL})$ & $8.9 \pm 5.5(37)$ & $10.3 \pm 7.5(37)$ & 0.38 \\
\hline Glucagon (pg/mL) & $164.6 \pm 33.5(37)$ & $171.6 \pm 42.4(37)$ & 0.43 \\
\hline $\begin{array}{l}\text { Systolic blood pres- } \\
\text { sure }(\mathrm{mmHg})\end{array}$ & $129.2 \pm 13.7(37)$ & $130.0 \pm 12.4(37)$ & 0.78 \\
\hline $\begin{array}{l}\text { Diastolic blood pres- } \\
\text { sure }(\mathrm{mmHg})\end{array}$ & $81.8 \pm 9.6(37)$ & $79.9 \pm 8.5(37)$ & 0.36 \\
\hline $\begin{array}{l}\text { Total cholesterol } \\
\text { (mg/dL) }\end{array}$ & $201.8 \pm 33.1(35)$ & $194.1 \pm 31.9(35)$ & 0.33 \\
\hline $\begin{array}{l}\text { HDL cholesterol } \\
\text { (mg/dL) }\end{array}$ & $50.9 \pm 9.0(35)$ & $53.4 \pm 15.4(35)$ & 0.42 \\
\hline $\begin{array}{l}\text { LDL cholesterol } \\
\qquad(\mathrm{mg} / \mathrm{dL})\end{array}$ & $109.6 \pm 33.2(35)$ & $96.1 \pm 25.0(35)$ & 0.06 \\
\hline
\end{tabular}

Table 1 continued

\begin{tabular}{|c|c|c|c|}
\hline Characteristics & $\begin{array}{l}\text { Dapagliflozin } \\
\text { group }\end{array}$ & $\begin{array}{l}\text { Metformin } \\
\text { group }\end{array}$ & $P$ value \\
\hline Triglyceride (mg/dL) & $142.8 \pm 53.1(37)$ & $145.2 \pm 69.0$ & 0.87 \\
\hline $\begin{array}{l}\text { Serum creatinine } \\
(\mathrm{mg} / \mathrm{dL})\end{array}$ & $0.7 \pm 0.1(37)$ & $0.7 \pm 0.2(37)$ & 0.72 \\
\hline Uric acid (mg/dL) & $5.6 \pm 1.1(37)$ & $5.8 \pm 1.2(37)$ & 0.41 \\
\hline Anti-diabetic drugs & $37(100.0)$ & $37(100.0)$ & - \\
\hline Biguanides & $37(100.0)$ & $37(100.0)$ & - \\
\hline DPP-4 inhibitors & $6(16.2)$ & $7(18.9)$ & 1.00 \\
\hline Sulfonylureas & $2(5.4)$ & $4(10.8)$ & 0.67 \\
\hline $\begin{array}{l}\text { a-Glucosidase } \\
\text { inhibitors }\end{array}$ & $2(5.4)$ & $1(2.7)$ & 1.00 \\
\hline Glinides & $2(5.4)$ & $4(10.8)$ & 0.67 \\
\hline Thiazolidinediones & $0(0.0)$ & $0(0.0)$ & - \\
\hline $\begin{array}{l}\text { Antihypertensive } \\
\text { drugs }\end{array}$ & $11(29.7)$ & $14(37.8)$ & 0.62 \\
\hline Diuretic drugs & $0(0.0)$ & $1(2.7)$ & 1.00 \\
\hline $\begin{array}{l}\text { Calcium channel } \\
\text { blockers }\end{array}$ & $6(16.2)$ & $10(27.0)$ & 0.40 \\
\hline ACE inhibitors & $4(10.8)$ & $0(0.0)$ & 0.11 \\
\hline $\begin{array}{l}\text { Angiotensin II } \\
\text { receptor block- } \\
\text { ers }\end{array}$ & $6(16.2)$ & $11(29.7)$ & 0.27 \\
\hline $\begin{array}{l}\text { Direct renin inhibi- } \\
\text { tors }\end{array}$ & $0(0.0)$ & $0(0.0)$ & - \\
\hline$\beta$-Blockers & $1(2.7)$ & $0(0.0)$ & 1.00 \\
\hline a-Blockers & $1(2.7)$ & $0(0.0)$ & 1.00 \\
\hline $\begin{array}{l}\text { Lipid-lowering } \\
\text { agents }\end{array}$ & $23(62.2)$ & $21(56.8)$ & 0.81 \\
\hline Statins & $14(37.8)$ & $15(40.5)$ & 1.00 \\
\hline Fibrates & $7(18.9)$ & $5(13.5)$ & 0.75 \\
\hline Ezetimibe & $2(5.4)$ & $1(2.7)$ & 1.00 \\
\hline Probucol & $1(2.7)$ & $0(0.0)$ & 1.00 \\
\hline EPAs & $3(8.1)$ & $3(8.1)$ & 1.00 \\
\hline Resins & $0(0.0)$ & $0(0.0)$ & - \\
\hline $\begin{array}{l}\text { Antithrombotic } \\
\text { agents }\end{array}$ & $1(2.7)$ & $1(2.7)$ & 1.00 \\
\hline Antiplatelet agents & $1(2.7)$ & $1(2.7)$ & 1.00 \\
\hline Anticoagulants & $0(0.0)$ & $0(0.0)$ & - \\
\hline
\end{tabular}

Data are number (\%), mean \pm standard deviation $(n)$, or median [first quartile, third quartile] ( $n)$. $P$ values by the $t$ test or Wilcoxon rank sum test for continuous data, and by Fisher exact test for categorical data

$B M I$ body mass index, UACR urinary albumin-to-creatinine ratio, $\mathrm{HbA1C}$ hemoglobin A1C, NGSP national glycohemoglobin standardization program, $H D L$ high-density lipoprotein, $L D L$ low-density lipoprotein, DPP-4 dipeptidyl peptidase-4, ACE angiotensin-converting enzyme, EPA eicosapentaenoic acid

\section{Adverse events}

Table 7 lists the reported/observed AEs during the study. The recorded AEs were six for the dapagliflozin group and nine for the metformin group. There was no significant difference in the incidence of AEs between the two groups. 
Table $2 \Delta$ FMD

\begin{tabular}{llll}
\hline & $\begin{array}{l}\text { Dapagliflozin } \\
\text { group }\end{array}$ & $\begin{array}{l}\text { Metformin } \\
\text { group }\end{array}$ & P value \\
\hline FMD (\%) & & & \\
FAS population & & & \\
Baseline & $4.80 \pm 1.86(37)$ & $5.37 \pm 2.95(37)$ & 0.33 \\
Week 16 & $5.66 \pm 2.12(37)$ & $5.18 \pm 2.09(37)$ & 0.33 \\
Change & $0.85 \pm 2.71(37)$ & $-0.19 \pm 2.51(37)$ & 0.09 \\
P value within & 0.06 & 0.65 & \\
$\quad$ group & & & \\
Subpopulation: HbA1c at baseline <7.0\% & & \\
Baseline & $4.72 \pm 1.88(24)$ & $4.88 \pm 2.76(20)$ & 0.83 \\
Week 16 & $5.47 \pm 2.42(24)$ & $5.33 \pm 2.23(20)$ & 0.85 \\
Change & $0.75 \pm 2.82(24)$ & $0.45 \pm 2.48(20)$ & 0.71 \\
$\begin{array}{l}\text { Pvalue within } \\
\text { group }\end{array}$ & 0.21 & 0.43 & \\
Subpopulation: HbA1c at baseline $\geq 7.0 \%$ & & \\
Baseline & $4.95 \pm 1.91(13)$ & $5.94 \pm 3.14(17)$ & 0.30 \\
Week 16 & $6.01 \pm 1.43(13)$ & $5.01 \pm 1.96(17)$ & 0.12 \\
Change & $1.05 \pm 2.59(13)$ & $-0.94 \pm 2.39(17)$ & 0.041 \\
$P$ value within & 0.17 & 0.13 & \\
$\quad$ group & & & \\
\hline
\end{tabular}

Data are mean \pm standard deviation $(n) . P$ values show results of comparisons between groups by $t$ test. $P$ values within groups are results of paired $t$ test FAS full analysis set. See Table 1 for other abbreviations
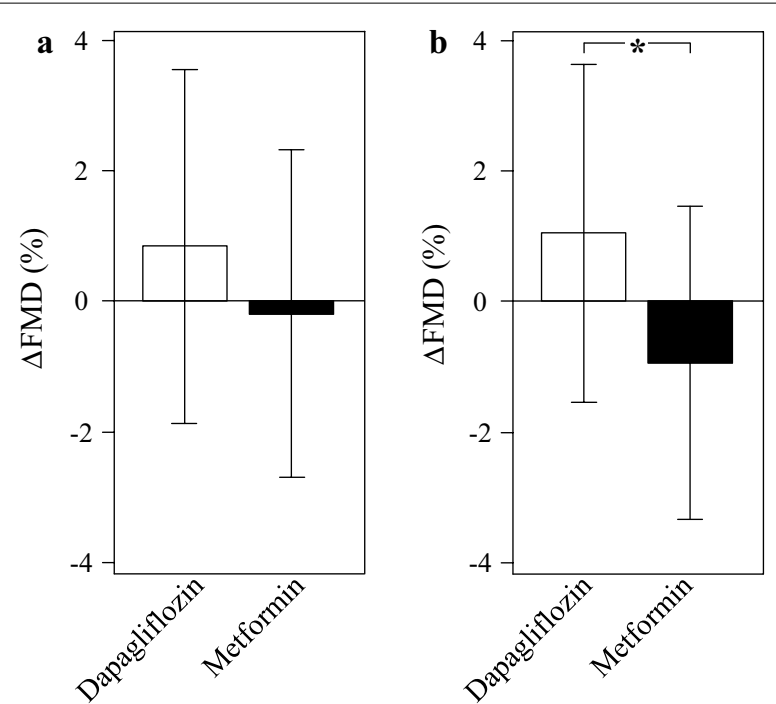

Fig. 2 Effects of each treatment on $\triangle F M D$. a $\triangle F M D$ in FAS. $\mathbf{b} \triangle F M D$ in subpopulation of $\mathrm{HbA} 1 \mathrm{c}$ at baseline $\geq 7.0 \%$. ${ }^{*} \mathrm{P}<0.05$

\section{Discussion}

Dapagliflozin is a relatively new oral glucose-lowering agent, and the DEFENCE study was designed to dissect the effects of dapagliflozin on endothelial function in T2DM patients with no history of cardiovascular diseases.
Table 3 Changes in parameters of glycemic control

\begin{tabular}{|c|c|c|c|}
\hline Parameters & $\begin{array}{l}\text { Dapagliflozin } \\
\text { group }\end{array}$ & $\begin{array}{l}\text { Metformin } \\
\text { group }\end{array}$ & $P$ value \\
\hline \multicolumn{4}{|l|}{$\mathrm{HbA1c}(\%)$} \\
\hline Baseline & $6.8 \pm 0.5(37)$ & $6.9 \pm 0.5(37)$ & 0.39 \\
\hline Week 16 & $6.5 \pm 0.5(37)$ & $6.5 \pm 0.6(37)$ & 0.76 \\
\hline Change & $-0.2 \pm 0.4(37)$ & $-0.4 \pm 0.3(37)$ & 0.09 \\
\hline $\begin{array}{l}P \text { value within } \\
\text { group }\end{array}$ & 0.001 & $<0.001$ & \\
\hline \multicolumn{4}{|c|}{$\mathrm{HbA1c}(\mathrm{mmol} / \mathrm{mol})$} \\
\hline Baseline & $50.3 \pm 5.5(37)$ & $51.4 \pm 5.7(37)$ & 0.39 \\
\hline Week 16 & $48.0 \pm 5.4(37)$ & $47.5 \pm 6.2(37)$ & 0.76 \\
\hline Change & $-2.4 \pm 4.2(37)$ & $-3.9 \pm 3.5(37)$ & 0.09 \\
\hline $\begin{array}{l}P \text { value within } \\
\text { group }\end{array}$ & 0.001 & $<0.001$ & \\
\hline \multicolumn{4}{|c|}{ Fasting plasma glucose (mg/dL) } \\
\hline Baseline & $133.5 \pm 27.1(37)$ & $139.6 \pm 20.2(37)$ & 0.28 \\
\hline Week 16 & $122.5 \pm 19.5(37)$ & $125.2 \pm 20.0(37)$ & 0.56 \\
\hline Change & $-11.0 \pm 20.2(37)$ & $-14.4 \pm 16.9(37)$ & 0.44 \\
\hline $\begin{array}{l}P \text { value within } \\
\text { group }\end{array}$ & 0.002 & $<0.001$ & \\
\hline \multicolumn{4}{|c|}{ C-peptide (ng/mL) } \\
\hline Baseline & $2.1 \pm 0.8(37)$ & $2.3 \pm 1.1(37)$ & 0.34 \\
\hline Week 16 & $2.0 \pm 0.8(37)$ & $2.1 \pm 1.0(37)$ & 0.65 \\
\hline Change & $-0.1 \pm 0.5(37)$ & $-0.2 \pm 0.5(37)$ & 0.29 \\
\hline $\begin{array}{l}P \text { value within } \\
\text { group }\end{array}$ & 0.27 & 0.007 & \\
\hline \multicolumn{4}{|l|}{ Insulin ( $\mu \mathrm{IU} / \mathrm{mL})$} \\
\hline Baseline & $8.9 \pm 5.5(37)$ & $10.3 \pm 7.5(37)$ & 0.38 \\
\hline Week 16 & $7.3 \pm 4.4(37)$ & $9.2 \pm 7.4(37)$ & 0.20 \\
\hline Change & $-1.6 \pm 3.7(37)$ & $-1.1 \pm 3.8(37)$ & 0.58 \\
\hline $\begin{array}{l}P \text { value within } \\
\text { group }\end{array}$ & 0.012 & 0.08 & \\
\hline \multicolumn{4}{|c|}{ Glucagon (pg/mL) } \\
\hline Baseline & $164.6 \pm 33.5(37)$ & $171.6 \pm 42.4(37)$ & 0.43 \\
\hline Week 16 & $152.9 \pm 24.5(37)$ & $159.3 \pm 36.8(37)$ & 0.39 \\
\hline Change & $-11.7 \pm 31.2(37)$ & $-12.3 \pm 31.6(37)$ & 0.93 \\
\hline $\begin{array}{l}P \text { value within } \\
\text { group }\end{array}$ & 0.029 & 0.023 & \\
\hline \multicolumn{4}{|l|}{ HOMA-IR } \\
\hline Baseline & $3.1 \pm 2.4(37)$ & $3.7 \pm 3.2(37)$ & 0.34 \\
\hline Week 16 & $2.3 \pm 1.5(37)$ & $3.0 \pm 3.0(37)$ & 0.16 \\
\hline Change & $-0.8 \pm 1.9(37)$ & $-0.7 \pm 1.5(37)$ & 0.73 \\
\hline $\begin{array}{l}P \text { value within } \\
\text { group }\end{array}$ & 0.010 & 0.009 & \\
\hline
\end{tabular}

Data are presented as mean \pm standard deviation $(n) . P$ values show results for comparisons between groups by $t$ test. $P$ values within groups are results of paired $t$ test

HOMA-IR homeostatic model assessment of insulin resistance. See Table 1 for other abbreviations

Several studies have already reported that the addition of dapagliflozin as monotherapy [24] or as an add-on to insulin $[24,25]$ was safe and effective in patients with 
Table 4 Changes in lipid parameters, markers of atherosclerosis, oxidative stress, and blood pressure

\begin{tabular}{|c|c|c|c|}
\hline Parameters & $\begin{array}{l}\text { Dapagliflozin } \\
\text { group }\end{array}$ & $\begin{array}{l}\text { Metformin } \\
\text { group }\end{array}$ & $\begin{array}{l}P \\
\text { value }\end{array}$ \\
\hline \multicolumn{4}{|c|}{ Total cholesterol (mg/dL) } \\
\hline Baseline & $201.8 \pm 33.1(35)$ & $194.1 \pm 31.9(34)$ & 0.33 \\
\hline Week 16 & $205.3 \pm 36.0(37)$ & $182.2 \pm 24.8(37)$ & 0.002 \\
\hline $\begin{array}{l}\text { Percentage } \\
\text { change (\%) }\end{array}$ & $2.2 \pm 13.4$ (35) & $-5.4 \pm 9.3(34)$ & 0.008 \\
\hline $\begin{array}{l}P \text { value within } \\
\text { group }\end{array}$ & 0.35 & 0.002 & \\
\hline \multicolumn{4}{|c|}{ HDL cholesterol (mg/dL) } \\
\hline Baseline & $50.9 \pm 9.0(35)$ & $53.4 \pm 15.4(34)$ & 0.42 \\
\hline Week 16 & $55.1 \pm 9.4(37)$ & $54.7 \pm 15.2(37)$ & 0.90 \\
\hline $\begin{array}{l}\text { Percentage } \\
\text { change (\%) }\end{array}$ & $8.1 \pm 13.4$ (35) & $3.4 \pm 15.3(34)$ & 0.18 \\
\hline $\begin{array}{l}P \text { value within } \\
\text { group }\end{array}$ & 0.001 & 0.20 & \\
\hline \multicolumn{4}{|c|}{ LDL cholesterol (mg/dL) } \\
\hline Baseline & $109.6 \pm 33.2(35)$ & $96.1 \pm 25.0(34)$ & 0.06 \\
\hline Week 16 & $124.0 \pm 33.6(37)$ & $102.7 \pm 26.0(37)$ & 0.003 \\
\hline $\begin{array}{l}\text { Percentage } \\
\text { change (\%) }\end{array}$ & $19.1 \pm 28.0(35)$ & $9.7 \pm 22.2(34)$ & 0.13 \\
\hline $\begin{array}{l}P \text { value within } \\
\text { group }\end{array}$ & $<0.001$ & 0.016 & \\
\hline \multicolumn{4}{|c|}{ Non-HDL cholesterol (mg/dL) } \\
\hline Baseline & $150.9 \pm 32.8(35)$ & $140.7 \pm 35.2(34)$ & 0.22 \\
\hline Week 16 & $150.2 \pm 33.1(37)$ & $127.4 \pm 24.7(37)$ & 0.001 \\
\hline $\begin{array}{l}\text { Percent change } \\
(\%)\end{array}$ & $0.9 \pm 17.0(35)$ & $-7.7 \pm 12.1$ (34) & 0.018 \\
\hline $\begin{array}{l}\text { P value within } \\
\text { group }\end{array}$ & 0.74 & $<0.001$ & \\
\hline \multicolumn{4}{|c|}{ Triglyceride (mg/dL) } \\
\hline Baseline & $142.8 \pm 53.1(37)$ & $145.2 \pm 69.0(37)$ & 0.87 \\
\hline Week 16 & $155.9 \pm 81.1(37)$ & $136.2 \pm 62.6(37)$ & 0.25 \\
\hline $\begin{array}{l}\text { Percentage } \\
\text { change (\%) }\end{array}$ & $11.3 \pm 40.8(37)$ & $4.2 \pm 53.4(37)$ & 0.53 \\
\hline $\begin{array}{l}P \text { value within } \\
\text { group }\end{array}$ & 0.10 & 0.63 & \\
\hline \multicolumn{4}{|c|}{ Adiponectin $(\mu \mathrm{g} / \mathrm{mL})$} \\
\hline Baseline & $2.6 \pm 1.8(37)$ & $2.7 \pm 1.8(37)$ & 0.77 \\
\hline Week 16 & $2.7 \pm 2.2(37)$ & $2.8 \pm 2.0(37)$ & 0.79 \\
\hline Change & $0.1 \pm 0.7(37)$ & $0.1 \pm 0.7(37)$ & 0.97 \\
\hline $\begin{array}{l}P \text { value within } \\
\text { group }\end{array}$ & 0.46 & 0.41 & \\
\hline \multicolumn{4}{|l|}{ ApoB48 $(\mu \mathrm{g} / \mathrm{mL})$} \\
\hline Baseline & $4.8 \pm 3.0(37)$ & $3.6 \pm 1.9(37)$ & 0.036 \\
\hline Week 16 & $5.1 \pm 3.9(37)$ & $3.1 \pm 1.8(37)$ & 0.008 \\
\hline Change & $0.3 \pm 2.8(37)$ & $-0.4 \pm 2.2(37)$ & 0.23 \\
\hline $\begin{array}{l}P \text { value within } \\
\text { group }\end{array}$ & 0.55 & 0.24 & \\
\hline \multicolumn{4}{|c|}{ Urinary 8-OHdG/creatinine (ng/mg Cre) } \\
\hline Baseline & $4.6 \pm 2.4(36)$ & $4.8 \pm 2.0(36)$ & 0.62 \\
\hline Week 16 & $4.0 \pm 1.9(37)$ & $5.8 \pm 2.3(37)$ & $<0.001$ \\
\hline Change & $-0.6 \pm 1.8(36)$ & $1.1 \pm 2.2(36)$ & $<0.001$ \\
\hline
\end{tabular}

Table 4 continued

\begin{tabular}{|c|c|c|c|}
\hline Parameters & $\begin{array}{l}\text { Dapagliflozin } \\
\text { group }\end{array}$ & $\begin{array}{l}\text { Metformin } \\
\text { group }\end{array}$ & $\begin{array}{l}P \\
\text { value }\end{array}$ \\
\hline $\begin{array}{l}P \text { value within } \\
\text { group }\end{array}$ & 0.047 & 0.004 & \\
\hline \multicolumn{4}{|c|}{ Systolic blood pressure (mmHg) } \\
\hline Baseline & $129.2 \pm 13.7(37)$ & $130.0 \pm 12.4(37)$ & 0.78 \\
\hline Week 16 & $126.4 \pm 13.2(37)$ & $130.3 \pm 13.5(37)$ & 0.21 \\
\hline Change & $-2.8 \pm 11.6(37)$ & $0.3 \pm 12.6(37)$ & 0.27 \\
\hline $\begin{array}{l}P \text { value within } \\
\text { group }\end{array}$ & 0.15 & 0.88 & \\
\hline \multicolumn{4}{|c|}{ Diastolic blood pressure (mmHg) } \\
\hline Baseline & $81.8 \pm 9.6(37)$ & $79.9 \pm 8.5(37)$ & 0.36 \\
\hline Week 16 & $80.0 \pm 8.1(37)$ & $79.1 \pm 7.5$ (37) & 0.61 \\
\hline Change & $-1.8 \pm 7.8(37)$ & $-0.8 \pm 5.8(37)$ & 0.52 \\
\hline $\begin{array}{l}P \text { value within } \\
\text { group }\end{array}$ & 0.16 & 0.41 & \\
\hline
\end{tabular}

Data are mean \pm standard deviation $(n)$, or median [first quartile, third quartile] (n). $P$ values show results for comparisons between groups by $t$ test or Wilcoxon rank sum test. $P$ values within groups are results of paired $t$ test

ApoB48 apolipoprotein B48, 8OHdG 8-hydroxy-2'-deoxyguanosin. See Table 1 for other abbreviations

inadequately controlled T2DM. Furthermore, not only the addition of dapagliflozin to metformin [26-28], but also initial combination therapy with both agents [29] resulted in improvement of glycemic control.

In this study, we planned to compare the effects of add-on dapagliflozin relative to those of metformin. Metformin is widely used worldwide and is known to provide protection against cardiovascular events [8]. A previous meta-analysis reported that there was no suggestion of increase risk for major adverse cardiovascular events with dapagliflozin compared with control [30]. However, to our knowledge, there is no published clinical trial that compared the effects of dapagliflozin add-on therapy to those of increased dose of metformin and little is known about the effects of the combination of dapagliflozin plus metformin on endothelial function. Thus, the DEFENCE study is the first study to address the effects of dapagliflozin on endothelial function as primary prevention of cardiovascular diseases.

$\mathrm{T} 2 \mathrm{DM}$ is one of the major risk factors for progression of atherosclerosis and development of cardiovascular diseases [1, 2]. Recently, the EMPA-REG OUTCOME study reported that empagliflozin, another SGLT2 inhibitor, reduced cardiovascular mortality and hospitalization for heart failure among patients with T2DM [17]. It is noteworthy that empagliflozin reduced the number of patients who required admission to the hospital for heart failure, but not nonfatal ischemic cardiovascular events [17]. In the EMPA-REG OUTCOME study, most of the enrolled patients had history of cardiovascular diseases, and reduction in the primary outcome was observed at 
Table 5 Changes in blood cell counts and uric acid

\begin{tabular}{|c|c|c|c|}
\hline Parameters & $\begin{array}{l}\text { Dapagliflozin } \\
\text { group }\end{array}$ & $\begin{array}{l}\text { Metformin } \\
\text { group }\end{array}$ & $P$ value \\
\hline \multicolumn{4}{|c|}{ White blood cell count $(/ \mu \mathrm{L})$} \\
\hline Baseline & $\begin{array}{l}6378.6 \pm 1553.5 \\
(36)\end{array}$ & $\begin{array}{l}6405.0 \pm 1507.6 \\
\quad(36)\end{array}$ & 0.94 \\
\hline Week 16 & $\begin{array}{l}6532.5 \pm 1646.0 \\
(35)\end{array}$ & $\begin{array}{l}6372.6 \pm 1700.5 \\
(34)\end{array}$ & 0.69 \\
\hline Change & $103.8 \pm 1166.7(34)$ & $\begin{array}{l}-106.2 \pm 1183.0 \\
(34)\end{array}$ & 0.46 \\
\hline $\begin{array}{l}P \text { value within } \\
\text { group }\end{array}$ & 0.61 & 0.60 & 0.95 \\
\hline \multicolumn{4}{|c|}{ Red blood cell count $\left(\times 10^{4} / \mu \mathrm{L}\right)$} \\
\hline Baseline & $468.9 \pm 40.2(36)$ & $468.3 \pm 41.8(36)$ & $<0.001$ \\
\hline Week 16 & $493.6 \pm 41.6(35)$ & $456.6 \pm 42.9(34)$ & $<0.001$ \\
\hline Change & $26.3 \pm 16.3(34)$ & $-12.0 \pm 17.5(34)$ & \\
\hline $\begin{array}{l}P \text { value within } \\
\text { group }\end{array}$ & $<0.001$ & $<0.001$ & \\
\hline \multicolumn{4}{|c|}{ Hemoglobin (g/dL) } \\
\hline Baseline & $14.3 \pm 1.3(36)$ & $14.4 \pm 1.3(36)$ & 0.70 \\
\hline Week 16 & $14.9 \pm 1.4(35)$ & $14.2 \pm 1.7(34)$ & 0.05 \\
\hline Change & $0.7 \pm 0.5(34)$ & $-0.2 \pm 0.9(34)$ & $<0.001$ \\
\hline $\begin{array}{l}P \text { value within } \\
\text { group }\end{array}$ & $<0.001$ & 0.21 & \\
\hline \multicolumn{4}{|l|}{ Hematocrit (\%) } \\
\hline Baseline & $42.6 \pm 3.6(36)$ & $42.7 \pm 3.3(36)$ & 0.94 \\
\hline Week 16 & $44.7 \pm 3.5(35)$ & $41.8 \pm 3.6(34)$ & 0.001 \\
\hline Change & $2.2 \pm 1.4(34)$ & $-0.9 \pm 1.8(34)$ & $<0.001$ \\
\hline $\begin{array}{l}P \text { value within } \\
\text { group }\end{array}$ & $<0.001$ & 0.006 & \\
\hline \multicolumn{4}{|c|}{ Platelet count $\left(\times 10^{4} / \mu \mathrm{L}\right)$} \\
\hline Baseline & $24.0 \pm 6.4(36)$ & $24.0 \pm 5.0(36)$ & 0.99 \\
\hline Week 16 & $23.9 \pm 6.3(35)$ & $24.5 \pm 4.7(34)$ & 0.64 \\
\hline Change & $-0.3 \pm 1.5(34)$ & $0.5 \pm 2.2(34)$ & 0.09 \\
\hline $\begin{array}{l}P \text { value within } \\
\text { group }\end{array}$ & 0.20 & 0.25 & \\
\hline \multicolumn{4}{|l|}{ Uric acid (mg/dL) } \\
\hline Baseline & $5.6 \pm 1.1(37)$ & $5.8 \pm 1.2(37)$ & 0.41 \\
\hline Week 16 & $4.9 \pm 1.1(36)$ & $5.9 \pm 1.1(36)$ & $<0.001$ \\
\hline Change & $-0.6 \pm 0.7(36)$ & $0.1 \pm 0.6$ & $<0.001$ \\
\hline $\begin{array}{l}P \text { value within } \\
\text { group }\end{array}$ & $<0.001$ & 0.43 & \\
\hline
\end{tabular}

Data are mean \pm standard deviation $(n) . P$ values show results of comparisons between groups by $t$ test. $P$ values within groups are results of paired $t$ test

3 months after the start of treatment with empagliflozin. Thus, it was concluded that the effect of empagliflozin on cardiovascular mortality is probably not related to the suppression of progression of atherosclerosis [17]. Another clinical trial; the DECLARE TIMI-58 study, which was designed to determine the effect of dapagliflozin on cardiovascular outcome, is ongoing but $40 \%$ of the enrolled patients had a previous cardiovascular event [31]. Thus, the efficacy of SGLT2 inhibitors as primary
Table 6 Changes in various parameters in patients with $\mathrm{HbA1c}$ at baseline of $\geq 7.0 \%$

\begin{tabular}{|c|c|c|c|}
\hline Parameters & $\begin{array}{l}\text { Dapagliflozin } \\
\text { group }\end{array}$ & $\begin{array}{l}\text { Metformin } \\
\text { group }\end{array}$ & $P$ value \\
\hline \multicolumn{4}{|c|}{ Urinary 8-OHdG/creatinine (ng/mg Cre) } \\
\hline Baseline & $4.3 \pm 1.7(13)$ & $4.4 \pm 2.0(17)$ & 0.88 \\
\hline Week 16 & $4.1 \pm 1.1(13)$ & $5.7 \pm 2.4(17)$ & 0.021 \\
\hline Change & $-0.2 \pm 1.6(13)$ & $1.3 \pm 2.3(17)$ & 0.042 \\
\hline $\begin{array}{l}P \text { value within } \\
\text { group }\end{array}$ & 0.66 & 0.031 & \\
\hline \multicolumn{4}{|c|}{ Red blood cell $\left(\times 10^{4} / \mu \mathrm{L}\right)$} \\
\hline Baseline & $485.6 \pm 32.2(13)$ & $463.5 \pm 41.4(16)$ & 0.12 \\
\hline Week 16 & $507.9 \pm 34.8(12)$ & $453.4 \pm 30.9(15)$ & $<0.001$ \\
\hline Change & $21.3 \pm 13.2(12)$ & $-14.7 \pm 18.1(15)$ & $<0.001$ \\
\hline $\begin{array}{l}P \text { value within } \\
\text { group }\end{array}$ & $<0.001$ & 0.007 & \\
\hline \multicolumn{4}{|c|}{ Hemoglobin (g/dL) } \\
\hline Baseline & $14.9 \pm 0.8(13)$ & $14.0 \pm 1.3(16)$ & 0.036 \\
\hline Week 16 & $15.6 \pm 0.7(12)$ & $14.0 \pm 1.9(15)$ & 0.008 \\
\hline Change & $0.7 \pm 0.4(12)$ & $-0.1 \pm 1.3(15)$ & 0.030 \\
\hline $\begin{array}{l}P \text { value within } \\
\text { group }\end{array}$ & $<0.001$ & 0.67 & \\
\hline \multicolumn{4}{|l|}{ Hematocrit (\%) } \\
\hline Baseline & $44.1 \pm 2.3(13)$ & $41.7 \pm 3.7(16)$ & 0.048 \\
\hline Week 16 & $46.1 \pm 2.0(12)$ & $40.7 \pm 3.0(15)$ & $<0.001$ \\
\hline Change & $2.0 \pm 1.3(12)$ & $-1.4 \pm 1.7(15)$ & $<0.001$ \\
\hline $\begin{array}{l}P \text { value within } \\
\text { group }\end{array}$ & $<0.001$ & 0.007 & \\
\hline \multicolumn{4}{|l|}{ Uric acid (mg/dL) } \\
\hline Baseline & $5.9 \pm 0.8(13)$ & $5.0 \pm 0.9(17)$ & 0.012 \\
\hline Week 16 & $5.2 \pm 0.6(12)$ & $5.3 \pm 1.0(17)$ & 0.74 \\
\hline Change & $-0.7 \pm 0.7(12)$ & $0.3 \pm 0.5(17)$ & $<0.001$ \\
\hline $\begin{array}{l}P \text { value within } \\
\text { group }\end{array}$ & 0.007 & 0.041 & \\
\hline
\end{tabular}

Data are mean \pm standard deviation $(n) . P$ values show results for comparisons between groups by $t$ test or Wilcoxon rank sum test. $P$ values within groups are results of paired $t$ test or Wilcoxon signed rank test

prevention of cardiovascular events and suppression of atherosclerosis remains unknown. In this regard, it is important to screen patients for the progression of atherosclerosis at an early stage in order to prevent cardiovascular events. Therefore, we set the inclusion criteria to include $\mathrm{HbA} 1 \mathrm{c} \geq 6.0$ and $<8.0 \%$ treated with only metformin $750 \mathrm{mg} /$ day or one type of oral glucose-lowering agent in addition to $750 \mathrm{mg} /$ day of metformin, and excluded patients with history of cardiovascular diseases. There is general agreement that vascular endothelial dysfunction is the initial stage of atherosclerosis, and considered the earliest predictor of future cardiovascular events in patients with T2DM [5]. Furthermore, FMD is recognized as a well-established surrogate marker of early endothelial dysfunction [32]. 
Table 7 Adverse events

\begin{tabular}{|c|c|c|c|}
\hline & $\begin{array}{l}\text { Dapagliflozin } \\
\text { group }(n=40)\end{array}$ & $\begin{array}{l}\text { Metformin } \\
\text { group }(n=40)\end{array}$ & $P$ value \\
\hline Any adverse events & $6(15.0)$ & $9(22.5)$ & 0.57 \\
\hline Hypoglycemia & $1(2.5)$ & $0(0.0)$ & 1.00 \\
\hline Allergic rhinitis & $0(0.0)$ & $1(2.5)$ & 1.00 \\
\hline Dry mouth & $1(2.5)$ & $0(0.0)$ & 1.00 \\
\hline $\begin{array}{l}\text { Esophageal varices } \\
\text { hemorrhage }\end{array}$ & $1(2.5)^{\mathrm{a}}$ & $0(0.0)$ & 1.00 \\
\hline Frequent urination & $1(2.5)$ & $0(0.0)$ & 1.00 \\
\hline $\begin{array}{l}\text { Upper respiratory } \\
\text { tract infection }\end{array}$ & $0(0.0)$ & $1(2.5)$ & 1.00 \\
\hline $\begin{array}{l}\text { Interstitial lung } \\
\text { disease }\end{array}$ & $1(2.5)^{\mathrm{a}}$ & $0(0.0)$ & 1.00 \\
\hline $\begin{array}{l}\text { Gastroesophageal } \\
\text { reflux disease }\end{array}$ & $1(2.5)$ & $0(0.0)$ & 1.00 \\
\hline Wound & $1(2.5)$ & $0(0.0)$ & 1.00 \\
\hline Pharyngitis & $0(0.0)$ & $1(2.5)$ & 1.00 \\
\hline Dyslipidemia & $1(2.5)$ & $0(0.0)$ & 1.00 \\
\hline $\begin{array}{l}\text { Decrease in appe- } \\
\text { tite }\end{array}$ & $0(0.0)$ & $1(2.5)$ & 1.00 \\
\hline Alopecia areata & $0(0.0)$ & $1(2.5)$ & 1.00 \\
\hline Palpitation & $0(0.0)$ & $1(2.5)$ & 1.00 \\
\hline Cervical dysplasia & $0(0.0)$ & $1(2.5)^{\mathrm{a}}$ & 1.00 \\
\hline $\begin{array}{l}\text { Lung cancer, } \\
\text { stage } 0\end{array}$ & $0(0.0)$ & $1(2.5)^{\mathrm{a}}$ & 1.00 \\
\hline Contusion & $0(0.0)$ & $1(2.5)$ & 1.00 \\
\hline Diarrhea & $0(0.0)$ & $1(2.5)$ & 1.00 \\
\hline
\end{tabular}

Data are from treated set population

$P$ values are results by Fisher exact test between groups

a Serious adverse event

In the present study, there was no significant difference in $\triangle$ FMD between the two groups in FAS population. However, dapagliflozin add-on therapy significantly improved $\triangle$ FMD in patients with $\mathrm{HbA} 1 \mathrm{c} \geq 7.0 \%$ compared to metformin-increased therapy. In the dapagliflozin group, $\triangle \mathrm{FMD}$ increased by $1.05 \pm 2.59 \%$ after 16 weeks of treatment. In this regard, it is reported that an increase of $1 \%$ in FMD is associated with $12 \%$ reduction in adjusted relative risk of future cardiovascular events [33]. These results indicate that dapagliflozin add-on therapy provides better protection of endothelial function and prevention of future cardiovascular events in early-stage T2DM patients with inadequate glycemic control, compared to metformin-increased therapy. Because there was no significant difference in glycemic control between the two groups, it seems that the additional benefit of dapagliflozin is mediated through mechanisms other than its glucose lowering effect. To elucidate such mechanisms, we evaluated certain putative biomarkers that are considered to be associated with the progression of atherosclerosis. Interestingly, urine $8-\mathrm{OHdG}$, a marker of oxidative stress, was significantly reduced only in the dapagliflozin group. This result suggests that dapagliflozin reduces oxidative stress and this might contribute to the observed improvement in endothelial function. In fact, previous studies reported that elevated $8-\mathrm{OHdG}$ was associated with progression of atherosclerosis [34] and diabetic vasculopathies [35]. In addition, experimental studies in rodents showed that SGLT2 inhibitors reduced urine 8-OHdG [36] and improved endothelial function [37]. These data support our hypothesis that dapagliflozin may improve endothelial function in T2DM by reducing oxidative stress. Interestingly, plasma uric acid was significantly reduced in the dapagliflozin group. Hyperuricemia can cause hypertension and vascular damage [38], and relates to cardiovascular diseases [39]. Thus, the observed decrease in serum uric acid could also play a role in the improvement of FMD in the dapagliflozin group. In addition, increases in red blood cell count, hemoglobin, and hematocrit were observed in the present study. SGLT2 inhibitors cause osmotic diuresis, and this contributes to changes in blood volume and blood cell kinetics [31]. Recently, increased hematocrit during SGLT2 inhibitors attracted attention as stimulus factor of erythropoiesis and oxygen transport to tissue as a protective role in cardiovascular diseases [40]. In this study, hematocrit was significantly increased in dapagliflozin group within not only FAS population analysis but also subgroup analysis of $\mathrm{HbA} 1 \mathrm{c}<7.0$ or $\geq 7.0 \%$. Interestingly, a covariance analysis for $\triangle F M D$ by taking the change of hematocrit into account did not show any significant difference in subgroup of $\mathrm{HbA} 1 \mathrm{c}<7.0$ and $\geq 7.0 \%$ ( $\mathrm{P}=0.51$ and 0.12 , respectively). These data suggest that increased hematocrit might play a role on the increased FMD at least in part in dapagliflozin group. However, the Hisayama study showed both elevated and decreased hematocrit were associated with increased cardiovascular diseases [41]. Thus, it is still not clear whether the effect of dapagliflozin on hematocrit contributed to the improvement of FMD. Other markers of atherosclerosis, such as LDL cholesterol, adiponectin, and apolipoprotein B48 were comparable between the two groups.

\section{Limitations}

Although these results clearly demonstrate the effectiveness of dapagliflozin add-on therapy, our study has several limitations. First, as this was an open-label design study, unexpected bias might occur because physicians knew their choice of treatment and subjects also knew the type of medications used. Second, the number of patients was relatively small and the duration of study was relatively short, so longer trials with larger sample size, preferably in subjects of different ethnicities, are needed. Third, subjects enrolled in this study had moderate hyperglycemia, so the effects of treatment in subjects with HbA1c of more than $8 \%$ remain unknown. Finally, we chose dapagliflozin $5 \mathrm{mg}$ but not $10 \mathrm{mg}$. The results of phase III trial of dapagliflozin 
in Japanese patients with T2DM reported that both doses of dapagliflozin produced significant reduction in $\mathrm{HbA1c}$ from baseline. The adjusted mean change in HbA1c from baseline to 24 week was almost the same level $(5 \mathrm{mg}$, $-0.41 \%$ and $10 \mathrm{mg},-0.45 \%$ ) [42]. In addition, another trial reported that dapagliflozin, initiated at $5 \mathrm{mg}$ once daily and titrated, as needed, to $10 \mathrm{mg}$ once daily, was well tolerated over 52 weeks in Japanese patients with T2DM [43]. The dose of dapagliflozin was $5 \mathrm{mg}$ for initial treatment and it was up-titrated to $10 \mathrm{mg}$ for the subjects whose HbA1c $>7.5 \%$ after 12 weeks [43]. In the present study, according to inclusion criteria, basal HbA1c level was $\geq 6.0$ and $<8.0 \%$, thus, the $5 \mathrm{mg}$ of dapagliflozin was considered to be enough and well tolerated. Furthermore, $5 \mathrm{mg}$ dapagliflozin costs a half price compared to $10 \mathrm{mg}$ dapagliflozin. Thus, in general, the standard dose of dapagliflozin is considered $5 \mathrm{mg}$ once daily in Japanese patients with T2DM.

\section{Conclusions}

The DEFENCE study is the first to evaluate the effects of dapagliflozin on vascular endothelial function in T2DM. Dapagliflozin add-on therapy on metformin improved endothelial function assessed by FMD in patients with inadequate glycemic control. So far, there is no solid evidence that SGLT2 inhibitors play a protective role on endothelial function or can suppress the progression of atherosclerosis. The results of this study suggest that the combination therapy of dapagliflozin and metformin is a potential therapeutic option for the primary prevention of cardiovascular disease in patients with early-stage T2DM and moderate hyperglycemia.

\section{Additional file}

Additional file 1. Table S1. Changes in body weight and BMI.

\section{Abbreviations \\ 8-OHdG: 8-hydroxy-2'-deoxyguanosin; DPP-4: dipeptidyl peptidase 4; FMD: flow-mediated dilation; SGLT2: sodium glucose cotransporter 2; T2DM: type 2 diabetes mellitus.}

\section{Authors' contributions}

$F S$, NK, and TH designed the study, reviewed the manuscript and contributed to the discussion. HU, MM and KI are responsible for patient enrollment and manuscript development. EK selected the statistical analysis methods. FS and NK wrote and edited the manuscript. The authors are fully responsible for all the content and editorial decisions, were involved in all the stages of the study and manuscript development. NK is the guarantor of this work and, as such, has full access to all study data and takes responsibility for the integrity of the data and accuracy of data analysis. All authors read and approved the final manuscript.

\section{Author details}

1 Division of Diabetes, Metabolism, and Endocrinology, Department of Medicine, Toho University Graduate School of Medicine, 6-11-1 Omori-Nishi, Ota-ku, Tokyo 143-8541, Japan. ${ }^{2}$ Department of Nephrology, Tokyo Kyosai Hospital, Tokyo, Japan. ${ }^{3}$ Life Science and Bioethics Research Center, Tokyo Medical and Dental University, Tokyo, Japan.

\section{Acknowledgements}

We thank Kenichi Tashiro (Haneda Bus-Road Clinic), Makiko Sasamoto (Sasamoto Medical Clinic), Takamasa Ichijyo (Saiseikai Yokohama Tobu Hospital), Mariko Higa (Saiseikai Yokohama Tobu Hospital), Yukie Fuse, Fukumi Yoshikawa, Hiroshi Yoshino, Masayo Ando, Shuki Usui, Momoko Kanaguchi, Ryo Iga, Mai Hijikata, Ken Kanazawa, Chie Fujita, Natsumi Mashima, Hirokazu Yamada, and Hiroki Takayama for the excellent technical assistance.

\section{Competing interests}

N. Kumashiro received lecture fees from Novo Nordisk Inc. T Hirose received research funds from AstraZeneca, Boehringer Ingelheim Pharmaceuticals, Inc., Astellas Pharma Inc., Ono Pharmaceutical Co., Ltd., Novo Nordisk Inc, Sanofi-Aventis Deutschland GmbH, Daiichi-Sankyo Co., Ltd., Eli Lilly Japan K.K., Takeda Pharmaceutical Company Limited, Mitsubishi Tanabe Pharma Corporation, Dainippon Sumitomo Pharma Co., Ltd., Kissei Pharmaceutical Co., Ltd., and Johnson \& Johnson, and received lecture fees from Sanofi-Aventis Deutschland GmbH, Eli Lilly Japan K.K., Novo Nordisk Inc, Takeda Pharmaceutical Company Limited, Daiichi-Sankyo Co., Ltd., Mitsubishi Tanabe Pharma Corporation, Merck \& Co., Inc, Dainippon Sumitomo Pharma Co., Ltd., Novartis Pharma K.K., Kissei Pharmaceutical Co., Ltd., Boehringer Ingelheim Pharmaceuticals, Inc., Ono Pharmaceutical Co., Ltd., and AstraZeneca. The funding agencies had no role in the study design, data collection and analysis, decision to publish, or preparation of the manuscript.

\section{Availability of data and materials}

The data analyzed during this study are available from the corresponding author of this article upon reasonable request.

\section{Ethics approval and consent to participate}

The study was approved by the Medical Ethics Committee of Toho University (approval \#27249) and was conducted according to the Declaration of Helsink and current legal regulations in Japan. All patients provided written informed consent before participation.

\section{Funding}

This study is supported by Astrazeneca Japan K.K. and ONO PHARMACEUTICAL CO., LTD; the manufacturer of dapagliflozin. None of the two pharmaceutical companies dictate or modify the results of the study.

\section{Publisher's Note}

Springer Nature remains neutral with regard to jurisdictional claims in published maps and institutional affiliations.

Received: 6 May 2017 Accepted: 20 June 2017

Published online: 06 July 2017

\section{References}

1. Kim JA, Montagnani M, Koh KK, Quon MJ. Reciprocal relationships between insulin resistance and endothelial dysfunction: molecular and pathophysiological mechanisms. Circulation. 2006;113(15):1888-904.

2. Fox CS, Coady S, Sorlie PD, Levy D, Meigs JB, D'Agostino RB Sr, Wilson PW, Savage PJ. Trends in cardiovascular complications of diabetes. JAMA. 2004;292(20):2495-9.

3. Haffner SM, Lehto S, Ronnemaa T, Pyorala K, Laakso M. Mortality from coronary heart disease in subjects with type 2 diabetes and in nondiabetic subjects with and without prior myocardial infarction. N Engl J Med. 1998;339(4):229-34.

4. Barr EL, Zimmet PZ, Welborn TA, Jolley D, Magliano DJ, Dunstan DW, Cameron AJ, Dwyer T, Taylor HR, Tonkin AM, et al. Risk of cardiovascular and all-cause mortality in individuals with diabetes mellitus, impaired fasting glucose, and impaired glucose tolerance: the Australian Diabetes, Obesity, and Lifestyle Study (AusDiab). Circulation. 2007;116(2):151-7.

5. Node K, Inoue T. Postprandial hyperglycemia as an etiological factor in vascular failure. Cardiovasc Diabetol. 2009;8:23.

6. Holman RR, Paul SK, Bethel MA, Matthews DR, Neil HA. 10-year followup of intensive glucose control in type 2 diabetes. N Engl J Med. 2008;359(15):1577-89. 
7. Ter Avest E, Stalenhoef AF, de Graaf J. What is the role of non-invasive measurements of atherosclerosis in individual cardiovascular risk prediction? Clin Sci. 2007;112(10):507-16.

8. UK Prospective Diabetes Study (UKPDS) Group. Effect of intensive bloodglucose control with metformin on complications in overweight patients with type 2 diabetes (UKPDS 34). Lancet. 1998;352(9131):854-65.

9. Consoli A, Gomis R, Halimi S, Home PD, Mehnert H, Strojek K, Van Gaal LF. Initiating oral glucose-lowering therapy with metformin in type 2 diabetic patients: an evidence-based strategy to reduce the burden of latedeveloping diabetes complications. Diabetes Metab. 2004;30(6):509-16.

10. Rojas LB, Gomes MB. Metformin: an old but still the best treatment for type 2 diabetes. Diabetol Metab Syndr. 2013;5(1):6.

11. Kalra S. Sodium glucose co-transporter-2 (SGLT2) inhibitors: a review of their basic and clinical pharmacology. Diabetes Ther. 2014;5(2):355-66.

12. Nauck MA, Del Prato S, Meier JJ, Duran-Garcia S, Rohwedder K, Elze M, Parikh SJ. Dapagliflozin versus glipizide as add-on therapy in patients with type 2 diabetes who have inadequate glycemic control with metformin: a randomized, 52-week, double-blind, active-controlled noninferiority trial. Diabetes Care. 2011;34(9):2015-22.

13. Majewski C, Bakris GL. Blood pressure reduction: an added benefit of sodium-glucose cotransporter 2 inhibitors in patients with type 2 diabetes. Diabetes Care. 2015;38(3):429-30.

14. Nauck MA. Update on developments with SGLT2 inhibitors in the management of type 2 diabetes. Drug Des Dev Ther. 2014;8:1335-80.

15. Cherney DZ, Perkins BA, Soleymanlou N, Har R, Fagan N, Johansen OE, Woerle HJ, von Eynatten M, Broedl UC. The effect of empagliflozin on arterial stiffness and heart rate variability in subjects with uncomplicated type 1 diabetes mellitus. Cardiovasc Diabetol. 2014;13:28.

16. Neal B, Perkovic V, de Zeeuw D, Mahaffey KW, Fulcher G, Stein P, Desai M, Shaw W, Jiang J, Vercruysse F, et al. Rationale, design, and baseline characteristics of the Canagliflozin Cardiovascular Assessment Study (CANVAS) — a randomized placebo-controlled trial. Am Heart J. 2013;166(2):217-23.

17. Zinman B, Wanner C, Lachin JM, Fitchett D, Bluhmki E, Hantel S, Mattheus M, Devins T, Johansen OE, Woerle HJ, et al. Empagliflozin, cardiovascular outcomes, and mortality in type 2 diabetes. N Engl J Med. 2015:373(22):2117-28

18. Singh JS, Fathi A, Vickneson K, Mordi I, Mohan M, Houston JG, Pearson ER, Struthers AD, Lang CC. Research into the effect Of SGLT2 inhibition on left ventricular remodelling in patients with heart failure and diabetes mellitus (REFORM) trial rationale and design. Cardiovasc Diabetol. 2016;15:97.

19. Corretti MC, Anderson TJ, Benjamin EJ, Celermajer D, Charbonneau F, Creager MA, Deanfield J, Drexler H, Gerhard-Herman M, Herrington D, et al. Guidelines for the ultrasound assessment of endothelial-dependent flowmediated vasodilation of the brachial artery: a report of the International Brachial Artery Reactivity Task Force. J Am Coll Cardiol. 2002;39(2):257-65.

20. Takeno K, Mita T, Nakayama S, Goto H, Komiya K, Abe H, Ikeda F, Shimizu T, Kanazawa A, Hirose T, et al. Masked hypertension, endothelial dysfunction, and arterial stiffness in type 2 diabetes mellitus: a pilot study. Am J Hypertens. 2012;25(2):165-70

21. Shigiyama F, Kumashiro N, Miyagi M, Iga R, Kobayashi Y, Kanda E, Uchino $H$, Hirose T. Linagliptin improves endothelial function in patients with type 2 diabetes: a randomized study of linagliptin effectiveness on endothelial function. J Diabetes Investig. 2017;8(3):330-40.

22. Suzuki K, Watanabe K, Suzuki T, Ouchi M, Futami-Suda S, Igari Y, Nakano $\mathrm{H}$, Oba K. Sitagliptin improves vascular endothelial function in Japanese type 2 diabetes patients without cardiovascular disease. J Diabetes Mellit. 2012;2:338-45.

23. Naka KK, Papathanassiou K, Bechlioulis A, Pappas K, Kazakos N, Kanioglou C, Kostoula A, Vezyraki P, Makriyiannis D, Tsatsoulis A, et al. Effects of pioglitazone and metformin on vascular endothelial function in patients with type 2 diabetes treated with sulfonylureas. Diabetes Vasc Dis Res. 2012;9(1):52-8.

24. Fioretto P, Giaccari A, Sesti G. Efficacy and safety of dapagliflozin, a sodium glucose cotransporter 2 (SGLT2) inhibitor, in diabetes mellitus. Cardiovasc Diabetol. 2015;14:142.

25. Sosale B, Sosale A, Bhattacharyya A. Clinical effectiveness and impact on insulin therapy cost after addition of dapagliflozin to patients with uncontrolled type 2 diabetes. Diabetes Ther. 2016;7(4):765-76.
26. Bailey CJ, Gross JL, Pieters A, Bastien A, List JF. Effect of dapagliflozin in patients with type 2 diabetes who have inadequate glycaemic control with metformin: a randomised, double-blind, placebo-controlled trial. Lancet. 2010;375(9733):2223-33.

27. Bolinder J, Ljunggren O, Johansson L, Wilding J, Langkilde AM, Sjostrom CD, Sugg J, Parikh S. Dapagliflozin maintains glycaemic control while reducing weight and body fat mass over 2 years in patients with type 2 diabetes mellitus inadequately controlled on metformin. Diabetes Obes Metab. 2014;16(2):159-69.

28. Bailey CJ, Gross JL, Hennicken D, lqbal N, Mansfield TA, List JF. Dapagliflozin add-on to metformin in type 2 diabetes inadequately controlled with metformin: a randomized, double-blind, placebo-controlled 102-week trial. BMC Med. 2013;11:43.

29. Bell KF, Katz A, Sheehan JJ. Quality measure attainment with dapagliflozin plus metformin extended-release as initial combination therapy in patients with type 2 diabetes: a post hoc pooled analysis of two clinical studies. Risk Manag Healthc Policy. 2016;9:231-41.

30. Sonesson C, Johansson PA, Johnsson E, Gause-Nilsson I. Cardiovascular effects of dapagliflozin in patients with type 2 diabetes and different risk categories: a meta-analysis. Cardiovasc Diabetol. 2016;15:37.

31. Abdul-Ghani M, Del Prato S, Chilton R, DeFronzo RA. SGLT2 inhibitors and cardiovascular risk: lessons learned from the EMPA-REG OUTCOME study. Diabetes Care. 2016;39(5):717-25.

32. Gokce N, Keaney JF Jr, Hunter LM, Watkins MT, Nedeljkovic ZS, Menzoian JO, Vita JA. Predictive value of noninvasively determined endothelial dysfunction for long-term cardiovascular events in patients with peripheral vascular disease. J Am Coll Cardiol. 2003;41(10):1769-75.

33. Matsuzawa Y, Kwon TG, Lennon RJ, Lerman LO, Lerman A. Prognostic value of flow-mediated vasodilation in brachial artery and fingertip artery for cardiovascular events: a systematic review and meta-analysis. J Am Heart Assoc. 2015;4(11):e002270.

34. Martinet W, Knaapen MW, De Meyer GR, Herman AG, Kockx MM. Elevated levels of oxidative DNA damage and DNA repair enzymes in human atherosclerotic plaques. Circulation. 2002;106(8):927-32.

35. Nishikawa T, Sasahara T, Kiritoshi S, Sonoda K, Senokuchi T, Matsuo T, Kukidome D, Wake N, Matsumura T, Miyamura N, et al. Evaluation of urinary 8-hydroxydeoxy-guanosine as a novel biomarker of macrovascular complications in type 2 diabetes. Diabetes Care. 2003;26(5):1507-12.

36. Ojima A, Matsui T, Nishino Y, Nakamura N, Yamagishi S. Empagliflozin, an inhibitor of sodium-glucose cotransporter 2 exerts anti-inflammatory and antifibrotic effects on experimental diabetic nephropathy partly by suppressing AGEs-receptor axis. Horm Metab Res. 2015;47(9):686-92.

37. Salim HM, Fukuda D, Yagi S, Soeki T, Shimabukuro M, Sata M. Glycemic control with ipragliflozin, a novel selective SGLT2 inhibitor, ameliorated endothelial dysfunction in streptozotocin-induced diabetic mouse. Front Cardiovasc Med. 2016;3:43.

38. Feig DI, Kang DH, Johnson RJ. Uric acid and cardiovascular risk. N Engl J Med. 2008:359(17):1811-21.

39. Odden MC, Amadu AR, Smit E, Lo L, Peralta CA. Uric acid levels, kidney function, and cardiovascular mortality in US adults: National Health and Nutrition Examination Survey (NHANES) 1988-1994 and 1999-2002. Am J Kidney Dis. 2014;64(4):550-7.

40. Ferrannini E, Mark M, Mayoux E. CV protection in the EMPA-REG OUTCOME trial: a "thrifty substrate" hypothesis. Diabetes Care. 2016;39(7):1108-14

41. Gotoh S, Hata J, Ninomiya T, Hirakawa Y, Nagata M, Mukai N, Fukuhara M, Ikeda F, Ago T, Kitazono T, et al. Hematocrit and the risk of cardiovascular disease in a Japanese community: The Hisayama Study. Atherosclerosis. 2015;242(1):199-204.

42. Kaku K, Kiyosue A, Inoue S, Ueda N, Tokudome T, Yang J, Langkilde AM. Efficacy and safety of dapagliflozin monotherapy in Japanese patients with type 2 diabetes inadequately controlled by diet and exercise. Diabetes Obes Metab. 2014;16(11):1102-10.

43. Kaku K, Maegawa H, Tanizawa Y, Kiyosue A, Ide Y, Tokudome T, Hoshino Y, Yang J, Langkilde AM. Dapagliflozin as monotherapy or combination therapy in Japanese patients with type 2 diabetes: an open-label study. Diabetes Ther. 2014;5(2):415-33. 